\title{
Medienpädagogik und Erwachsenenbildung
}

Annäherungen an ein schwieriges Verhältnis

Vortrag im Rahmen des Medienpädagogischen Kolloquiums an der Pädagogischen Hochschule Ludwigsburg am 11.12.2003

Bevor ich mich nun dem Verhältnis "Medienpädagogik und Erwachsenenbildung" zuwende, möchte ich mich zunächst herzlich bei Prof. Horst Niesyto bedanken, der mich zu diesem Vortrag und dem anschließenden Workshop eingeladen hat. Für mich ist es immer eine besondere Herausforderung, die Ergebnisse und Konzepte, die wir in unseren Projekten und Forschungsarbeiten im Deutschen Institut für Erwachsenenbildung (DIE) erarbeiten, in den Hochschuldiskurs ein zu bringen.

Das Verhältnis von Medienpädagogik und Erwachsenenbildung ist ein wechselseitiges. Auf der einen Seite haben wir das Verhältnis der Disziplin Erwachsenenbildung - und damit eines Ausschnitts der Pädagogik - zur Medienpädagogik, auf der anderen Seite das Verhältnis der Medienpädagogik zur Erwachsenenbildung. Beides sind keine ganz unproblematischen Verhältnisse. Darauf werde ich im Rahmen meines Vortrags eingehen.

Gleichzeitig möchte ich mich auch von der Betrachtung dieses Verhältnisses lösen und die Bedeutung vor allem der Neuen Medien für die Erwachsenenbildung und deren Auswirkungen beleuchten. Außerdem möchte ich Ihnen noch ein paar Perspektiven für den Diskurs eröffnen.

Mein Vortrag wird folgende Gliederung haben:

1. Einführung

2. Diskurse und Trends in der Erwachsenenbildung

3. Medienpädagogik und Erwachsenenbildung

4. Neue Medien in der Erwachsenenbildung

5. Perspektiven

\section{Einführung}

\subsection{Zur Arbeit des Deutschen Instituts für Erwachsenenbildung}

Das Deutsche Institut für Erwachsenenbildung (DIE) ist ein Institut der Leibniz-Gemeinschaft und wird von Bund- und Ländern finanziert. Es ist das zentrale Forum für Wissenschaft und Praxis der Weiterbildung in Deutschland. Als wissenschaftliches Serviceinstitut vermittelt es zwischen 
Forschung und Praxis der Erwachsenenbildung, liefert Grundlagen für eine praxisorientierte Forschung und entwickelt innovative Konzepte. Dienstleistung, Entwicklungsforschung, Vernetzung und internationale Aktivitäten sind die Eckpfeiler der Institutsarbeit. Die Serviceleistungen des DIE richten sich an professionelle Weiterbildner/innen und Wissenschaftler/innen im Bereich Weiterbildung.

Das DIE arbeitet seit Mitte dieses Jahres in Programmstrukturen, d.h. in zeitlich befristeten Themenfeldern, die sich an den zentralen Dimensionen der Weiterbildung orientieren. Dies sind:

$>$ Lernen Erwachsener,

> Professionelles Handeln in der Weiterbildung,

$>$ Organisationen der Weiterbildung und

$>$ System der Weiterbildung.

Daneben gibt es zum einen die Verwaltung und zum anderen einen Bereich der "Zentralen Wissenschaftlichen Dienste", der die Bibliothek, den Internet-Service, das Lektorat, die Veranstaltungsorganisation und die Öffentlichkeitsarbeit umfasst. Ein auf drei Jahre befristeter Projektschwerpunkt beschäftigt sich mit dem Aufbau eines "Europäischen Weiterbildungskollegs".

Innerhalb der Programmschwerpunkte wird zum einen an Produkten gearbeitet, die sich auf aktuelle Themen der Weiterbildung und Anforderungen aus Forschung und Praxis beziehen, zum anderen werden Drittmittelprojekte realisiert, die konkrete Fragestellungen in den Blick nehmen, wie z.B.:

$>$ Innovative Ansätze in der Grundbildung durch medienbasierte Zugänge (@Ipha)

$>$ Bildungspass unter besonderer Berücksichtigung informellen Lernens (BIBER)

$>$ Wissenschaftliche Begleitung des BMBF-Programms "Lernende Regionen - Förderung von Netzwerken" (LernReg)

$>$ Qualitätstestierung in der Weiterbildung und

$>$ Service Institutionenberatung zur Öffnung für neue Lernkulturen und Beratung bei neuen Angebotsformen (SELBER).

Meine Aufgaben im DIE sind vielfältig. Ich Medienbeauftragter des DIE und Sprecher der Institutskonferenz, dem zentrale Beratungsgremium der Wissenschaftler/innen. Ich arbeite als Wissenschaftler im Programm "Organisationen der Weiterbildung" und beschäftige mich derzeit mit Fragen des Benchmarking, der Vernetzung und des Wissens- und Innovationsmanagement in Kultur- und Weiterbildungseinrichtungen. In diesem Jahr habe ich eine größere Forschungsarbeit zum Thema "Neue Medien und Organisation in Weiterbildungseinrichtungen" abgeschlossen, aus der ich Ihnen später einige Ergebnisse vorstellen werde.

Doch möchte ich zunächst auf die Begriffskontexte Erwachsenenbildung/ Weiterbildung eingehen. 


\subsection{Begriffe Erwachsenenbildung/Weiterbildung}

Die Diskussion um die Klärung der Begriffe Weiterbildung bzw. Erwachsenenbildung ist spätestens seit dem Strukturplan des Deutschen Bildungsrats von 1970 virulent (Deutscher Bildungsrat 1970), in dem Erwachsenenbildung/Weiterbildung als vierte Säule des Bildungssystems beschrieben wurden. Zwar werden heute die Begriffe weitgehend synonym gebraucht, doch zeigt unter anderem die Diskussion im Kontext der Reflexionen über das Erziehungssystem im Rahmen systemtheoretischer Überlegungen (vgl. Lenzen/Luhmann 1997), dass in Anbetracht der pluralen Struktur und der zunehmenden "Entgrenzung" von Weiterbildung (vgl. Kade 1997) eine theoretisch untermauerte Begriffsbestimmung überfällig ist.

Eine begriffliche Trennung von Weiterbildung und Erwachsenenbildung, wie sie zum Beispiel von Koring (1999:10) vorgenommen wird, ist heute bei vielen Autor/inn/en nicht mehr zu finden (vgl. z.B. Peters 1999: 169; Faulstich et al. 1992: 9). Weinberg (2000: 10f.) weist darauf hin, dass durch die Einführung des Begriffs "Weiterbildung" durch den Deutschen Bildungsrat dieser zu einem Bildungssystembegriff wurde, der die gleichrangig nebeneinander stehenden Begriffe "berufliche Weiterbildung" und "allgemeine Erwachsenenbildung" umfasst. Weinberg verwendet "Erwachsenenbildung" als Sammelbegriff für Weiterbildung, nicht-institutionalisierte Erwachsenenbildung und informelles Erwachsenenlernen (Weinberg 2000: 39).

Neben der Einordnung der Begriffe im Bildungssystem ist es für uns wichtig - wenn wir uns dem Feld Erwachsenenbildung/Weiterbildung nähern uns das Funktionssystem näher anzuschauen.

\subsection{Funktionssystem Erwachsenenbildung/Weiterbildung}

Das Funktionssystem Erwachsenenbildung/Weiterbildung lässt sich auf unterschiedlichen Ebenen beschreiben. Zum einen haben wir die Trägerebene. Nach Faulstich et al. umfasst die Weiterbildung "öffentliche, private, betriebliche, berufliche, politische und allgemeinbildende Lehr- und Lernveranstaltungen für Erwachsene" (1992: 9). Diese werden von einer Vielzahl von Einrichtungen mit völlig unterschiedlicher Zielrichtung, Rechtsform, Trägerstruktur und Arbeitsweise angeboten (vgl. Nuissl 2001: 86). Faulstich (1997: 64) beschreibt vier spezifische Profile von Weiterbildungsinstitutionen, die sich aus der Zwischenstellung zwischen Staat, Interessensorganisationen und privaten Unternehmen ergeben, sich aber nicht vollständig voneinander trennen lassen:

$>$ öffentliche Erwachsenenbildungsträger,

$>$ partikulare Erwachsenenbildungsträger mit ihren Funktionen für gesellschaftliche Großgruppen,

$>$ kommerzielle Weiterbildungsunternehmen,

$>$ Bildungsabteilungen in Unternehmen.

Eine andere Möglichkeit der Systematisierung liefert die Beschreibung von Bezugssystemen. So weist Kade auf die zunehmende Entgrenzung und 
Entstrukturierung der Weiterbildung hin, wenn konstatiert wird, dass Bildungs- und Lernprozesse Erwachsener heute „in großer Zahl außerhalb pädagogischer, d.h. auf Bildung und Lernen spezialisierter Institutionen und jenseits professioneller Betreuung" stattfinden (1997: 19). Prof. Mader und ich haben den Versuch einer Systematisierung im Hinblick auf Zugänge zu Wissen in institutionalisierten und außerinstitutionellen Kontexten unternommen (vgl. Mader/Stang 2001). Wir unterscheiden zwischen wissensbasierten Dienstleistungen, zu denen auch die Angebote der institutionalisierten Weiterbildung gehören, und wissenslatenten Kontexten, die Informations- und Wissensoptionen in alltäglichen Lebenssituationen beschreiben, wie sie uns auch die Medien bieten.

Eine weitere Beschreibung des Funktionssystems Weiterbildung liefert Schäffter. Er unterscheidet drei "Operationskreise“, bei denen es sich um "geschlossene Kontextierungen mit je spezifischen Relevanzmustern von Bildung und Lernen“ handelt (Schäffter 1998: 108), die jeweils eine spezifische Entwicklungslogik und Entwicklungsgeschichte haben:

> Operationskreis I: Ordnungspolitischer Entscheidungsrahmen

$>$ Operationskreis II: Weiterbildungseinrichtung

$>$ Operationskreis III: Aneignungsstrukturen und Lernmilieus.

Diese Systematisierung bezieht sich auf Weiterbildungseinrichtungen als ein zentrales strukturelles Element. Schäffter betrachtet Weiterbildungseinrichtungen unter einer systemischen Perspektive und weist darauf hin, dass sie sich nicht auf den betrieblichen Aspekt beschränken lassen, sie "sind als strukturierter Zusammenhang zwischen den drei in sich geschlossenen, aber "locker verkoppelten' Operationskreisen zu fassen" (Schäffter 1998: 106). Er versteht Institutionalisierung im Operationskreis I ordnungspolitisch, in Operationskreis II erhält sie die eingeschränkte Bedeutung von betriebsförmigen Organisationsstrukturen und im Operationskreis III kommt sie „im Sinne biographischer und milieuspezifischer Strukturierungen der Bildungsaneignung zum Ausdruck" (Schäffter 1998: 114).

Sie sehen, welche Ebenen des Systems

Erwachsenenbildung/Weiterbildung wir zu berücksichtigen haben, wenn wir uns dem Themenfeld Medienpädagogik und Erwachsenenbildung nähern. Doch bevor ich dies tue, erscheint es mir wichtig, sich kurz die aktuellen Diskurse und Trends in der Erwachsenenbildung anzuschauen auch wenn dies für Sie keine Neuland darstellt. Aber vielleicht eröffnet es neue Perspektiven, wenn wir uns später dem Verhältnis von Medienpädagogik und Erwachsenenbildung nähern.

\section{Diskurse und Trends in der Erwachsenenbildung}

Ich werde versuchen in aller Kürze einige Diskurse und Trends der letzten Jahre zu bündeln. Folgende Themen möchte ich dabei ansprechen:

$>$ Lebenslanges, selbstgesteuertes und informelles Lernen,

$>$ Gestaltung von Lehr-/Lernarrangements, 
$>$ Finanzierung und Weiterbildungsmarkt,

$>$ Organisation und Management,

$>$ Qualität,

$>$ Professionalität,

$>$ Marketing und Öffentlichkeitsarbeit,

Vernetzung/Netzwerke.

\subsection{Lebenslanges, selbstgesteuertes und informelles Lernen}

Die Diskussionen über lebenslanges, selbstgesteuertes und nicht zuletzt informelles Lernen haben in den letzten Jahren zu einem Perspektivenwechsel in der Weiterbildung geführt. Seit den 1970er Jahren hat die internationale Diskussion über das "lifelong learning", die vor allem in den organisatorischen Kontexten der UNESCO, der OECD und der EU geführt wurde, den Blick auf die Tatsache gelenkt, dass in Anbetracht permanenter gesellschaftlicher Veränderungen alle Menschen ständig lernen müssen (vgl. Dohmen 2001).

Für die Weiterbildung und ihre Institutionen hatte die Diskussion insofern Folgen, als zum einen auf der organisatorischen Ebene über veränderte Lernorte und Lernarrangements nachgedacht wurde, zum anderen methodische Prinzipien wie die Selbstorganisation der Lernprozesse verstärkt in den Blick genommen wurden (vgl. Kraus 2001).

Auch das Thema "selbstgesteuertes Lernen“ wurde im Zusammenhang mit der Diskussion über das lebenslange Lernen seit Mitte der 1990er Jahre verhältnismäßig spät in der deutschen Weiterbildung aufgegriffen (vgl. Arnold 2001; Faulstich 1998). Faulstich verweist darauf, dass mit dem Begriff "selbstgesteuertes Lernen" auch immer ein "anti-institutioneller Affekt" mitschwingt (1998: 11). Das dürfte auch einer der Gründe dafür gewesen sein, dass die Weiterbildungspraxis sich erst zögerlich mit den institutionellen Konsequenzen der Förderung des selbstgesteuerten Lernens beschäftigt hat. Inzwischen werden allerdings in den Weiterbildungseinrichtungen vielfältige Konzepte entwickelt, um selbstgesteuertes Lernen zu unterstützen (dazu ausführlich: Dietrich 2001).

Ein weiterer Diskurskontext in diesem Zusammenhang dreht sich um das „informelle Lernen“. Auch hier wurde in Deutschland eine Diskussion aus dem angelsächsischen Bildungsdiskurs aufgegriffen. Unter „informellem Lernen" wird sowohl das unbeabsichtigte und beiläufige Lernen als auch ein bewusstes Lernen außerhalb organisierter Bildungsangebote verstanden (vgl. Dohmen 2001: 18ff.). Allerdings fehlt es bislang an einer theoretischen Durchdringung dieses Begriffs und vor allem an einer Analyse der Konsequenzen für organisierte Bildungsangebote.

Im Zusammenhang dieser Diskurse wird immer wieder auf die Bedeutung der Neuen Medien hingewiesen (vgl. u.a. Stang et al. 2001). Es geht dabei sowohl um didaktisch-methodische Dimensionen des Lernens mit Me- 
dien als auch um organisatorische Dimensionen in Bezug auf Weiterbildungseinrichtungen.

\subsection{Gestaltung von Lehr-/Lernarrangements}

Wenn heute über "Lernen" gesprochen wird, werden damit nicht nur institutionalisierte Bildungskontexte wie Schule, Ausbildung, Hochschule und Weiterbildung in Verbindung gebracht, sondern - wie eben gezeigt - auch vielfältige Formen des außerinstitutionellen Lernens. Die Begriffsvielfalt von "Lernarrangements", "Lernsettings", "Lernkulturen" und "Lernarchitekturen" ist nur ein Ausdruck für die "Pluralisierung des Lehrens und Lernens" (vgl. Nuissl et al. 1997), die eine Suchbewegung beschreibt, den gesellschaftlichen Anforderungen mit veränderten Lehr-/Lernkonzepten Rechnung zu tragen.

Die Hauptaufgabe für die Weiterbildung und ihre Institutionen ist die Gestaltung von Lernoptionen und damit von Lehr-/Lernarrangements, d.h. von didaktisch-methodisch konzipierten Angeboten. Im Zusammenhang mit der Diskussion um das lebenslange Lernen hat sich in den letzten Jahren der Blick erneut dafür geweitet, wie sich Lehr-/Lernarrangements auch außerhalb traditioneller Kursstrukturen gestalten lassen (vgl. Stang $2001 \mathrm{~b})$. Heute werden unter anderem multi- und telemediale Lernumgebungen (vgl. z.B. Kerres 1998), aber auch Kultureinrichtungen, wie zum Beispiel Bibliotheken (vgl. Umlauf 2001) und Museen (vgl. Behrens et al. 2001), als Lernorte immer mehr in den Blick genommen.

Eine der zentralen Herausforderungen für die Weiterbildungseinrichtungen stellt die Weiterentwicklung der Konzepte für Lehr-/Lernarrangements dar. Damit verbunden wird der weitere Umbau der Institutionen zu Serviceeinrichtungen rund um die Weiterbildung sein (vgl. Stang 1998). Die Neuen Medien werden dabei sowohl bei der Gestaltung von Lehr/Lernarrangements als auch bei der Gestaltung von medienbasierten Serviceangeboten eine zunehmende Bedeutung erhalten.

\subsection{Finanzierung und Weiterbildungsmarkt}

Zwar forderte der Deutsche Bildungsrat 1970 die Übernahme der öffentlichen Verantwortung für die Weiterbildung, doch kam es nie zu einer übergreifenden staatlichen Regelung bzgl. dieser "vierten Säule“ des Bildungssystems (vgl. Strunk 2000: 319ff.). Vielmehr entstand ein Ordnungsmodell der Staatssubsidiarität, das einen Rahmen für sehr unterschiedliche Anbieter in öffentlicher, freier und privater Trägerschaft bildete (vgl. Meisel 2001a: 216). So verschieden sich die Trägerstruktur darstellt, so unterschiedlich sind auch die Quellen, aus denen sich deren Finanzierung speist. Neben Entgelten der Teilnehmenden sind dies Förderungen durch Betriebe, die Betriebsangehörigen die Teilnahme bezahlen oder selbst Angebote gestalten, Mitgliedsbeiträge und Zuwendungen von gesellschaftlichen Organisationen (z.B. Kirchen) sowie staatliche Zuwendungen von Gebietskörperschaften wie Bund, Länder und Kommunen (vgl. Nuissl 1999: 399; Rohlmann 2001: 119). 
Eine intensive Analyse von Finanzierungsstrukturen der Weiterbildung als explizites Thema der Weiterbildungsdiskussion blieb bis in die 1980er Jahre die Ausnahme (vgl. Nuissl 1999: 398f.). Unter anderem durch die Krise der öffentlichen Haushalte in den 1980er Jahren und die damit verbundenen Auswirkungen auf die öffentlich geförderten Weiterbildungseinrichtungen (vgl. Bayer 1998: 21; Strunk 2000: 321) rückte das Thema "Finanzierung" immer mehr in den Blickpunkt des Diskurses über Weiterbildung. Unter dem Diktat der Wirtschaftlichkeit ${ }^{1}$ wurden Effizienz und Effektivität von Weiterbildungseinrichtungen oft nur noch unter der Kostenperspektive und kaum mehr unter der Nutzenperspektive betrachtet.

Der "Weiterbildungsmarkt" wurde Anfang der 1990er Jahre zu einem wichtigen Bezugspunkt der Diskussionen über die Zukunft der Weiterbildung (vgl. z.B. Friebel et al. 1993; Nuissl 1995). Meisel (2001: 217) hält den Marktbegriff allerdings für problematisch, da die Vielfalt der Akteure mit ihren unterschiedlichen Bedingungen dazu führt, dass die Teilbereiche der Weiterbildung nach jeweils "unterschiedlichen Modalitäten“ funktionieren. Dessen ungeachtet hat in der Weiterbildungspraxis, neben der finanziellen Situation und der zunehmenden Konkurrenz, die Diskussion über den Weiterbildungsmarkt bei vielen Weiterbildungseinrichtungen dazu geführt, die eigene Organisationsstruktur in den Blick zu nehmen und Veränderungsprozesse in Gang zu setzen. In der Forschung wurden ab Mitte der 1990er Jahre verstärkt lokale und regionale "Weiterbildungsmärkte“ zum Objekt umfangreicher Analysen (vgl. z.B. Dröll 1999; Körber et al. 1995; Tippelt et al. 1996).

Darüber hinaus wurde in Deutschland Ende der 1990er Jahre damit begonnen, im Kontext internationaler Diskussionen über neue Formen der Finanzierung von Weiterbildung nachzudenken und veränderte ordnungspolitische Konzepte zu entwickeln (vgl. z.B. Balzer/Nuissl 2000; Ehmann 2001).

\subsection{Organisation und Management}

Die erhöhten Anforderungen, Weiterbildungseinrichtungen als Organisationen weiterzuentwickeln, erfordern vom (Leitungs-)Personal besonders zwei Kompetenzen: erwachsenenpädagogische Fachkompetenz und Managementkompetenz (vgl. Geißler 1998: 82). Dass diese Kompetenzen in Zeiten struktureller Veränderungen von besonderer Relevanz sind, liegt auf der Hand. Die gesellschaftlichen Modernisierungsprozesse fordern die Weiterbildungseinrichtungen in ihrer gesamten Struktur und Organisation heraus. Es kann deshalb nicht verwundern, dass seit Anfang/Mitte der 1990er Jahre das Thema "Organisationsentwicklung” immer mehr in die Diskurse und die Alltagspraxis der Weiterbildung Einzug gehalten hat.

Mit der zunehmenden Aufmerksamkeit für Fragen der Organisation ging auch eine verstärkte Auseinandersetzung mit Fragen des Managements einher (vgl. Meisel 2001b). Management umfasst in diesem Zusammenhang Tätigkeiten wie planen, organisieren, anleiten und kontrollieren (vgl.

\footnotetext{
1 Einen guten Überblick zum Thema Wirtschaftlichkeit in Weiterbildungseinrichtungen liefert: Meisel et al. 1998.
} 
Nuissl 1998: 12), die dazu beitragen, (Weiterbildungs-)Einrichtungen zu lenken und systematisch weiterzuentwickeln. Zu den Managementaufgaben innerhalb einer Weiterbildungseinrichtung sind zum Beispiel Konzeptentwicklung, Organisation, Marketing, Reflexion, Führung und Evaluation zu zählen (vgl. Schläfli 2001: 213). Dabei werden in den Diskursen zwei Ebenen des Managements in einer Weiterbildungseinrichtungen unterschieden (vgl. z.B. Meisel 1999a: 430f.):

$>$ die Leitungsebene, auf der vor allem das strategische und normative Management für die Gesamteinrichtung anfällt,

$>$ die Ebene des hauptberuflich pädagogischen Personals, auf der das Planen und Betreuen von Lehr-/Lernarrangements zu verorten ist.

Eine Ebene ist allerdings bislang im Diskurs über Weiterbildungsmanagement noch nicht im ausreichenden Maße berücksichtigt worden,

$>$ die Ebene der technischen Betreuung, auf der die Planung und Weiterentwicklung der technischen Infrastruktur zur zentralen Managementaufgabe gehört.

Diese dritte Ebene erweist sich besonders in Anbetracht der zunehmenden Technisierung von Weiterbildungseinrichtungen (z.B. Internetanbindung, Intranet oder Veranstaltungsinfrastruktur) als wichtiges Managementproblem, für das es bislang an konzeptionellen Eckpunkten fehlt.

\subsection{Qualität}

Unter anderem im Zusammenhang mit der zunehmenden Ökonomisierung der Weiterbildung und den damit verbundenen Diskursen über Finanzierung, Wirtschaftlichkeit und Weiterbildungsmarkt entwickelte sich ebenfalls Anfang/Mitte der 1990er Jahre eine intensive Auseinandersetzung über das Thema "Qualität in der Weiterbildung". Dabei geht es in der Weiterbildung um eine spezifische Art der Qualität: um Qualität verbunden mit dem inhaltlichen Ziel Bildung.

Diese Perspektive ist vor allem deshalb wichtig, weil in den Anfängen der intensiveren Qualitätsdiskussion ${ }^{2}$ eine Orientierung hin auf die ISO9000ff.-Norm festzustellen war, die nur die Prozessqualität prüft, nicht aber die Produktqualität, also u.a. die pädagogische Professionalität (vgl. Krug 1999: 20f.). Die Auseinandersetzung mit der ISO-Norm führte zwar zu einer starken Verunsicherung in der Weiterbildung, wurde aber als Chance genutzt, andere Qualitätskonzepte in den Blick zu nehmen. Dabei entwickelte sich ein breites Spektrum von Möglichkeiten, das es den Weiterbildungseinrichtungen erlaubt, jene auszuwählen, die am besten den jeweiligen Bedürfnissen entspricht (vgl. Gnahs 1999: 17). Die Palette reicht von Konzepten der Selbstevaluation bis zu Evaluationen durch externe Gutachter. Konzepte wie ISO 9000ff. finden sich genauso in der Weiterbildungslandschaft wie Ansätze des Total Quality Management

2 Die Qualitätsdebatte ist in der Weiterbildung immer wieder vor allem auch unter der Perspektive der Professionalisierung geführt worden (vgl. Meisel 2001b: 107ff.). Insofern handelt es sich nicht um eine neue Debatte, doch wurde sie Anfang/Mitte der 1990er Jahre deutlich intensiviert. 
(TQM), des Benchmarking-Verfahrens EFQM, einzelne Gütesiegel etc. (vgl. Krug 1999).

Die Qualitätsdebatte der letzten zehn Jahre hat dazu geführt, dass Selbstverständigungsprozesse darüber stattgefunden haben, was die spezifische Qualität von Weiterbildungseinrichtungen ausmacht und wie Qualitätsmanagement realisiert werden kann (vgl. Meisel 1999b). Dabei haben sich übergreifend folgende Qualitätsebenen herauskristallisiert, die zu berücksichtigen sind (vgl. u.a. Faulstich 1991; Landesverband 1996; Stang 1999):

$>$ Einrichtungsqualität (u.a. Organisationsstruktur, Personal, Unterrichtsräume, Kommunikation und Kooperation),

> Programmqualität (u.a. Konzeption, curriculare Planung, Information),

$>$ Durchführungsqualität (Kompetenz der Dozent/innen, Lernprozess, Evaluation),

$>$ Erfolgsqualität (Effektivität, Zufriedenheit, Qualitätssicherung).

Entscheidend für Qualität in der Weiterbildung ist letztendlich jedoch, wie sich Ansprüche der Weiterbildungsanbieter mit den Bedürfnisses der an Bildung Teilnehmenden treffen (vgl. Ehses/Zech 1999: 19).

Betrachtet man diese Ebenen, lässt sich leicht erkennen, in welchem Maße die Diskurse über Qualität auch für den Einsatz Neuer Medien in Weiterbildungseinrichtungen von Relevanz sind. Allerdings bleibt festzustellen, dass dieses Themenfeld bislang nur unzureichend in der Qualitätsdebatte berücksichtigt wurde.

\subsection{Professionalität}

Eng mit dem Thema Qualitätssicherung in der Weiterbildung hängt auch das Thema Professionalisierung zusammen. Siebert (1999: 77) merkt im Rückblick auf die Geschichte der Weiterbildung an, dass sich zwar die Verberuflichung im Laufe der Jahre fortgesetzt, dass sich die Professionalisierung im Sinne eines unverwechselbaren Berufsbilds aber noch nicht ausgeprägt hat. Deshalb erstaunt es nicht, dass die Frage der Professionalisierung in der Weiterbildung nach wie vor zu den zentralen Diskursfeldern gehört.

Professionalität im Bereich der Weiterbildung bezieht sich für Ehses und Zech (1999: 16ff.) auf drei "Rationalitäten“: die betriebswirtschaftliche, die pädagogische sowie die organisationale. Sie beschreiben die Funktionen der hauptberuflich pädagogischen Mitarbeiter/innen (HPM) bezogen auf die drei Ebenen als Finanzmanager, Bildungsmanager und Organisationsmanager. Das heißt, Professionalität in der Weiterbildung konzentriert sich eben nicht mehr nur auf das pädagogische Bezugssystem. Dies ist vor allem auch vor dem Hintergrund von Bedeutung, dass sich in den letzten Jahren die Organisation von Bildung und die Durchführung von Bildung zunehmend "professionell" ausdifferenziert haben. Zumindest in großen Weiterbildungseinrichtungen ist der betriebswirtschaftliche Kompetenzbereich dem Leitungspersonal, der organisationale dem hauptberuflich pädagogischen Personal und der pädagogische - im direkten Kontakt mit den 
Teilnehmenden - den meist freiberuflichen bzw. nebenberuflichen Kursleitenden zuzuordnen.

Betrachten wir das Feld der Entwicklungen im Bereich der Neuen Medien, wird deutlich, dass bislang in dem Diskurs über Professionalität in der Weiterbildung der Kompetenzbereich Technik nicht als tragendes Element diskutiert wird. Dies stellt ein Defizit dar, denn die Organisation und das Management der technischen Infrastruktur werden in Zukunft an Bedeutung zunehmen, und es fehlt an orientierenden Beschreibungen des dafür notwendigen Kompetenzprofils von Mitarbeitenden. Dieses unterscheidet sich durch die besonderen Anforderungen, die die Betreuung der technischen Infrastruktur in Weiterbildungseinrichtungen darstellt, von üblichen Profilen der EDV-Organisation.

\subsection{Marketing und Öffentlichkeitsarbeit}

Die bereits beschriebene Ökonomisierung des Weiterbildungsbereichs sowie die Krise in der Finanzierung haben auch dazu geführt, dass Themen wie "Marketing" und "Öffentlichkeitsarbeit" seit Anfang/Mitte der 1990er Jahre verstärkt in den Diskurs über Weiterbildung Einzug gehalten haben (vgl. Meisel 1994).

Marketing beschäftigt sich auf der Basis von Marktforschung bzw. Marktanalysen mit der Gestaltung von Produkten und deren Preisen, der Werbung und der Distribution. Da es in der Weiterbildung nicht um den Verkauf eines eindeutig definierten Produktes geht, orientiert sich das Weiterbildungsmarketing an dem Konzept des Non-Profit-Marketing, dessen Ausrichtung mehr auf die Veränderung von Verhaltensweisen zielt, indem nicht nur Produkte, sondern auch Ideen vermarktet werden (vgl. Meisel 2001c: 215). Das bedeutet, dass es sich bei Weiterbildungsmarketing um eine zentrale Strategie zur Weiterentwicklung der Institution handelt.

Öffentlichkeitsarbeit ist ein Teil des Marketings, der in der Weiterbildung in den letzten Jahren einen immensen konzeptionellen Schub erhalten hat. Eine der zentralen Grundlagen von Öffentlichkeitsarbeit ist es, dass sich die Einrichtungen über ihre Institutionsidentität, ihr Leitbild im Klaren sind. Die Diskussion über die Corporate Identity (vgl. Nuissl/Rein 1997) ist also eng mit dem Thema Öffentlichkeitsarbeit verbunden. Dieses wurde vor allem ab Anfang der 1990er Jahre besonders virulent, da durch die ökonomischen Rahmenbedingungen die Marktpositionierung der einzelnen Weiterbildungseinrichtungen größere Bedeutung bekam, als dies vorher der Fall war, sich die Angebotspalette immer stärker ausdifferenzierte und damit Kommunikation des Angebots an Bedeutung zunahm.

In den letzten Jahren hat durch die Entwicklung der Neuen Medien deren Bedeutung für das Marketing und die Öffentlichkeitsarbeit immens zugenommen, nicht nur auf der Ebene der Produktion von Materialien zur Öffentlichkeitsarbeit (vgl. Rein 2001: 242), sondern in zunehmenden Maße auch auf der Ebene der Distribution. Immer mehr Weiterbildungseinrichtungen verfügen über eine eigene Homepage im World Wide Web, die als Portal für Informationen, Anmeldung und teilweise auch Bildungsmaterialien zur Verfügung gestellt wird. 1997 waren fast 300 von ca. 1.000 Volkshochschulen mit einer Homepage im Netz vertreten (vgl. Wolpert 
1998: 105), im Mai 2002 waren in der Adressdatei zur VHS-Statistik 622 Homepages angegeben, heute sind es 700 .

\subsection{Vernetzung/Netzwerke}

Die Vernetzung von Bildungseinrichtungen ist vor allem im Kontext des Förderschwerpunkts des BMBF "Lernende Regionen“ zu einem wichtigen Thema in der Weiterbildung geworden. Doch auch schon vorher gab es vielfältige Ansätze z.B. Bildungs- und Kultureinrichtungen zu vernetzen, um Synergieeffekte bei der Entwicklung von Bildungsangeboten für die Bevölkerung zu erzielen (vgl. Stang 2002).

Die finanziellen Rahmenbedingungen sind ein weiterer Grund dafür, dass die "Vernetzung" im Sinne der Zusammenlegung von Institutionen betrieben wird, was allerdings nur bedingt zu Erfolgen führen kann. Wichtiger ist vielmehr unser gesamtes Bildungssystem unter der Perspektive der Vernetzung neu zu denken. Es gilt Modelle in den Blick zu nehmen, die neben Kultur- und Weiterbildungseinrichtungen, auch Schulen und Hochschulen vernetzen. Die Bedeutung der Neuen Medien als Informations- und Kommunikationsmedium wird hier in den nächsten Jahren zunehmen.

Bevor ich nun auf eines der Diskurs- und Trendthemen - die Neuen Medien - ausführlicher eingehe, möchte ich an dieser Stelle, einige Anmerkungen zum Verhältnis von Medienpädagogik und Erwachsenenbildung machen.

\section{Medienpädagogik und Erwachsenenbildung}

\subsection{Traditionen des Mediendiskurses in der Erwachsenenbildung}

Technikbasierte Medien spielen in der Erwachsenenbildung von jeher eine besondere Rolle. Ob die Kinematographie, der Hörfunk oder das Fernsehen, die Auseinandersetzung mit den jeweils "neuen“ Medien und die Integration in Bildungsangebote waren immer kennzeichnend für Erwachsenenbildung (vgl. Wittpoth 2001). Mit Medienverbundprojekten und der Entwicklung von medienunterstützten Selbstlernzentren wurde bereits in den 1970er Jahren der Versuch unternommen, neue Formen von Lehr/Lernarrangements zu gestalten. Allerdings konnten sich diese Konzepte in der Erwachsenenbildung kaum durchsetzen (vgl. Schäfer 2001: 59ff.).

Die Neuen Medien sind spätestens seit der intensiven Diskussion über die gesellschaftlichen Auswirkungen der Informations- und Kommunikationstechniken Anfang der 1980er Jahre (vgl. Kubicek/Rolf 1985) auch ein Thema in der Erwachsenenbildung (vgl. Bohn et al. 1987; Petsch et al. 1989). Neben Aktivitäten im Zusammenhang mit "Offenen Kanälen“ und "Bürgerradios", die von den Entwicklungen in den Kabelpilotprojekten ausgingen (vgl. Baacke et al. 1990), waren es vor allem Angebote im Umgang mit dem Computer, die in die Weiterbildung Einzug hielten. Das 
Thema Informationstechnik wurde in Form von Angeboten von nahezu allen Weiterbildungsträgern aufgegriffen, wie eine umfassende Erhebung der Kontaktstelle für wissenschaftliche, künstlerische und berufliche Weiterbildung der Gesamthochschule Kassel Mitte der 1980er Jahre zeigte (vgl. Faulstich et al. 1985). Allerdings handelte es sich dabei in den meisten Fällen um Anpassungsfortbildungen (vgl. Seidel/Mikus 1987: 40).

Diese Struktur hat sich bis heute nur wenig verändert. Nach wie vor liegen zum Beispiel im Bereich der Volkshochschulen über $90 \%$ der Angebote im Programmbereich "Arbeit - Beruf" (vgl. Stang 2003: 140 ff.).

Der Begriff der Medienkompetenz wie er von Baacke entwickelt wurde mit seinen Dimensionen Medienkritik, Medienkunde, Mediennutzung und Mediengestaltung - spielte in der Erwachsenenbildung vor allem in den 1970er und Anfang der 1980er Jahre eine wichtige Rolle. Heute sind es wie gezeigt - in der Hauptsache EDV-Schulungen, die in den Blick geraten, wenn man sich Medienangebote in der Erwachsenenbildung anschaut.

Obwohl der kompetente Umgang mit den Neuen Medien zu einer zentralen Herausforderung geworden ist; d.h., dass neben den technischen Fertigkeiten, die Kompetenzen der Nutzung der Neuen Medien zur Kommunikation und kreativen Gestaltung sowie die Fähigkeit zur kritischen Beurteilung der Möglichkeiten und Grenzen der Neuen Medien entwickelt werden müssen, ist diese Thematik in der Erwachsenenbildung nur unzureichend aufgegriffen worden. Erst durch Diskussionen rund um das E-Learning hat auch wieder der Mediendiskurs in die Erwachsenenbildung Einzug gehalten.

Im Forschungsmemorandum für die Erwachsenen- und Weiterbildung, das im Jahr 2000 von der Sektion Erwachsenenbildung der Deutschen Gesellschaft für Erziehungswissenschaften veröffentlicht wurde, spielen die Medien eine unterordnete Rolle. Folgende fünf Forschungsfelder sind dort ausgewiesen (vgl. Arnold et al. 2002):

$>$ Lernen Erwachsener,

$>$ Wissensstrukturen und Kompetenzbedarfe,

> Professionelles Handeln,

$>$ Institutionalisierung,

$>$ System und Politik.

Doch nur in zwei Bereichen wird ein Medienbezug hergestellt. Im Forschungsfeld "Lernen Erwachsener" bezieht sich ein Schwerpunkt auf "Lernen in virtuellen Umwelten und leiblichen Gebundenheiten", im Forschungsfeld "Professionelles Handeln" bezieht sich ein Schwerpunkt auf "Medienumgang und Mediengestaltung". Auf den medienpädagogischen Diskurs wird dabei nicht eingegangen.

Die Medienpädagogik selbst hat in der Erwachsenenbildung in den letzten Jahren wenn, dann nur durch einzelne Initiativen u.a. im Rahmen des DIE als Bezugspunkt gedient. Medienpädagogik in der Erwachsenenbildung bedarf einer neuen Fundierung. Dies gilt allerdings auch in der umgekehrten Richtung. 


\subsection{Bedeutung von Erwachsenenbildung in der Medienpädagogik}

Lassen Sie mich bitte nochmals zu Dieter Baacke zurückkommen und seine Definition von Medienpädagogik:

"Medienpädagogik umfasst alle sozialpädagogischen, sozialpolitischen und sozialkulturellen Überlegungen und Maßnahmen sowie Angebote für Kinder, Jugendliche und Erwachsene, die ihre kulturellen Interessen und Entfaltungsmöglichkeiten, ihre persönlichen Wachstums- und Entwicklungschancen sowie ihre sozialen und politischen Ausdrucks- und Partizipationschancen betreffen, sei es als einzelne, als Gruppe oder als Organisationen und Institutionen" (Baacke 1997: S. 5).

Baacke nimmt hier das gesamte Bildungsspektrum in Zuständigkeitsbereich von Medienpädagogik. Doch im medienpädagogischen Diskurs der letzten Jahre ist es nicht gelungen, die Erwachsenenbildung zu einem zentralen Themenfeld zu machen. Betrachtet man sich z.B. die Aktivitäten der Gesellschaft für Medienpädagogik und Kommunikationskultur oder der Kommission Medienpädagogik der Deutschen Gesellschaft für Erziehungswissenschaften wird deutlich, dass sich die Diskurs vor allem auf die Schule, die außerschulische Jugendbildung und die Hochschule beziehen. Dies mag daran liegen, dass Medienpädagogik über Jahre vor allem im schulischen und außerschulischen Kontext seine Praxis- und Forschungsbezüge hatte.

Ich hoffe, dass ich mit diesen kurzen Beschreibungen deutlich machen konnte, warum ich im Untertitel meines Vortrags von einem schwierigen Verhältnis von Medienpädagogik und Erwachsenenbildung gesprochen habe. Im Folgenden möchte ich mich der Thematik auf der inhaltlichen Ebene zuwenden und mich anhand des Themenfeldes "Neue Medien“ dem Mediendiskurs in der Erwachsenenbildung nähern.

\section{Neue Medien in der Erwachsenenbildung}

\subsection{Zur Bedeutung Neuer Medien in der Erwachsenenbildung}

Die Diskurse über die Bedeutung der Neuen Medien in der Weiterbildung haben sich durch verschiedene technische Entwicklungen bis hin zu Multimedia und Internet in den letzten zehn Jahren intensiviert und verändert (vgl. Nispel et al. 1998a und 1998b; Stang et al. 1999). Die Auswirkungen der Neuen Medien sind auf den unterschiedlichsten Ebenen der Weiterbildung zu beobachten. Auf der einen Seite gibt es Bemühungen, medienbezogene Neuerungen im didaktischen Design und in der methodischen Präsentation von Bildungsinhalten zu entwickeln (vgl. Klimsa 1993), auf der anderen Seite bieten die Neuen Medien veränderte Optionen im organisatorischen Kontext von Verwaltung, Öffentlichkeitsarbeit usw. in den Weiterbildungseinrichtungen.

Bei der genaueren Betrachtung der Diskurse geraten besonders vier Ebenen in den Blick, auf denen eine Auseinandersetzung mit den Verände- 
rungspotenzialen durch die Neuen Medien Relevanz erhält (vgl. Kerres 1998; Stang 2001a: 20ff.):

$>$ die inhaltliche Ebene von Angeboten,

> die Gestaltung von Lehr-/Lernarrangements,

$>$ die Rolle der Lehrenden,

$>$ die Rolle der Institutionen.

Unter einer inhaltlichen Perspektive rückt die Vermittlung von Medienkompetenz in Anbetracht der Problematik des "Digital Divide" in den Blick (vgl. Rein 1996; Stang et al. 2001). Auf der Ebene der Lehr-/Lernarrangements geht es um die Frage, wie Neue Medien z.B. in Form von Lernsoftware oder E-Learning in sinnvolle didaktisch-methodisch gestaltete Bildungskonzepte integriert und wie neue medienadäquate Formen des Lehrens und Lernens etabliert werden können (vgl. Kerres 1998). Die Frage, wie sich die Rolle der Lehrenden durch die Neuen Medien verändert und welche neuen Professionselemente benötigt werden, lässt sich heute noch nicht abschließend beantworten. Doch der Ausbau von TelelearningAngeboten zeigt, dass das Teletutoring eine wichtige Kompetenz für die Zukunft sein wird (vgl. Bruns/Gajewski 1999). In den letzten Jahren hat sich gezeigt, dass die Veränderungen durch die Neuen Medien im Kontext der Weiterbildung vielschichtige Auswirkungen nicht nur auf Lehr-/Lernarrangements, sondern auch auf die Weiterbildungseinrichtungen insgesamt haben werden (vgl. Hagedorn 1998a und 1998b; Stang 1998).

Besonders intensiv werden die Diskussionen um eine veränderte Lernkultur derzeit unter dem Schlagwort „E-Learning“ geführt. Darunter werden inzwischen alle computergestützten Entwicklungen auf der Lehr-/LernEbene subsumiert (vgl. Stang 2001d). Obwohl viele Studien nachzuweisen versuchen, dass dem E-Learning die Zukunft gehört (vgl. z.B. McGovern 2001), gibt es doch in den letzten beiden Jahren auch verstärkt kritische Einschätzungen, was den Einsatz der Neuen Medien für das Lehren und Lernen betrifft. Nicht nur, dass auf dem zunächst boomenden Markt des E-Learning inzwischen einige Firmen Insolvenz anmelden mussten, auch die pädagogische Qualität vieler E-Learning-Angebote wird problematisiert. Köllinger (2001) hat in einer Marktstudie den Entwicklungsstand und die Trends des E-LearningMarktes in Deutschland beschrieben und darauf hingewiesen, dass nur wenige der verfügbaren Angebote bereits die Potenziale medienbasierten Lernens ausschöpfen.

Viele der heute agierenden E-Learning-Anbieter konzentrieren sich auf die Entwicklung von Lernplattformen für Großfirmen, die diese für die betriebliche Weiterbildung nutzen. Doch werden dort per E-Learning vor allem klassische Computer-Themen vermittelt. In einer Studie der Firma „unicmind.com “ ${ }^{3}$ wird die Rangliste der Themen angeführt von Office-Software $(66 \%)$, es folgen andere Anwendungssoftware (44\%) und Bedienung von Betriebssystemen (38\%).

\footnotetext{
3 Informationen unter: http://www.unicmind.com/elearningstudie.pdf (Stand: 12.08.2003).
} 
Auch die Volkshochschulen haben damit begonnen, neben den traditionellen Kursen E-Learning-Angebote in Form von Telelernen zu etablieren. Eine Bestandsaufnahme zum "Telelernen an Volkshochschulen" (Rudolf 2001: 82ff.) ergab für 200115 Volkshochschulen, die Telelern-Angebote haben, und 42, die Telelern-Angebote planen. Außerdem haben sich seit 2000 bundesweite Telelern-Plattformen für Volkshochschulen etabliert, ${ }^{4}$ die aber nur sehr begrenzte Angebote präsentiert.

Neben dem Telelernen spielen die Neuen Medien im unterstützenden Einsatz von traditionellen Kursangeboten eine zunehmende Rolle. So gibt es zum Beispiel inzwischen eine Fülle von Sprachlernprogrammen, die didaktisch-methodisch in die Kurskonzepte integriert werden können (vgl. Zeidler 2001). Inzwischen wird für die Integration von Präsenz- und Telelernen der Begriff "Blended Learning“ als neues Schlagwort verwendet.

Für den Bereich der Verwaltung in Weiterbildungseinrichtungen wurde bereits in den 1980er Jahren auf die Bedeutung der Neuen Medien hingewiesen (vgl. Eckert et al. 1983). Durch neue Verwaltungsprogramme, Öffentlichkeitsarbeit im Internet bis hin zu der Möglichkeit der Online-Anmeldung für Bildungsangebote wurden die Neuen Medien als Arbeitsinstrument in den Weiterbildungseinrichtungen immer wichtiger.

Betrachtet man die bislang vorgestellten Diskurse und Trends, lassen sich unter anderem folgende Ebenen in der Weiterbildung ausmachen, auf denen die Neuen Medien zum Tragen kommen:

$>$ als Arbeits-, Informations- und Kommunikationsinstrument im Büround Verwaltungsalltag (z.B. für internes Wissensmanagement, Online-Anmeldungen),

$>$ als Mittel für Marketing und Öffentlichkeitsarbeit (z.B. via Internet),

$>$ als Thema und Inhalt von Bildungsangeboten,

$>$ als unterstützendes Unterrichtsmittel,

$>$ als Instrument zur Gestaltung neuer Lehr-/Lernarrangements (z.B. computergestütztes Selbstlernzentrum, Online-Lernen, mediengestützte Kursbegleitung).

Insgesamt spielen die Neuen Medien, wie gezeigt, auf den unterschiedlichen Ebenen in der Weiterbildung eine besondere Rolle. Im Folgenden möchte ich exemplarisch die Bereiche medienbasierte Lehr/Lernarrangements, Organisation und Professionsprofile etwas ausführlicher beleuchten.

\subsection{Medienbasierte Lehr-/Lernarrangements}

Wenn wir uns medienbasierte Lehr-/Lernarrangements anschauen, können wir im wesentlichen folgende unterscheiden:

Medieneinsatz in Präsenzkursen

$>$ Sideline-Angebote

\footnotetext{
4 Informationen unter: http://www.vhs-virtuell.de (Stand: 28.01.2004), http://www.vhs-kampus.de (Stand: 28.01.2004), http://www.e-vhs-akademie.de (Stand: 28.01.2004).
} 
$>$ Lernsoftware

$>$ E-Learning

$>$ Blended Learning

$>$ Virtual/Augmented Reality

\section{Medieneinsatz in Präsenzkursen}

Der Medieneinsatz in Präsenzkursen gestaltet sich sehr unterschiedlich. Er reicht vom Einsatz mediengestützter Präsentationsformen z.B. mit Hilfe von Computer und Datenbeamer bis hin zur Nutzung des Internets für die inhaltliche Recherche.

\section{Sideline-Angebote}

Die Unterstützung von Präsenzangeboten durch aufbereitete Ressourcen im Internet, die nur für die Teilnehmenden zugänglich sind, wird im amerikanischen mit "Sideline Courses" bezeichnet. Solche Unterstützungsangebote im Internet erweitern die didaktisch-methodischen Möglichkeiten.

\section{Lernsoftware}

Die Palette von Lernsoftware ist breit (vgl. Stang 2001). Folgende Arten von Lernsoftware lassen sich unterscheiden:

$>$ Multimediale Informationssysteme

$>$ Trainings- und Übungsprogramme

$>$ Tutorielle Programme

$>$ Simulationen.

Ich möchte an dieser Stelle gar nicht so sehr auf didaktisch-methodische Konzeptionen eingehen, sondern Ihnen lieber zwei exemplarische Angebote vorstellen.

$>$ "Die Kunst des Präsentierens" der Firma Nitor

$>$ "Target Guest Service" der Firma Learning Sciences Corporation in Chicago/USA

Sie sehen es gibt unterschiedliche Formen der Gestaltung von Lernsoftware. Ich möchte hier nicht auf die Vor- und Nachteile eingehen, möchte aber zumindest darauf hinweisen, dass sich verschiedene Lernsoftware auch sehr unterschiedlich in didaktisch gestaltete Lehr-/Lernkontexte einbetten lässt.

\section{E-Learning}

Zu E-Learning, das heute im wesentlichen mit Online-Lernen gleichgesetzt wird, möchte ich nur soviel sagen, dass es in den letzten Jahren eine intensive Debatte über den pädagogischen "Mehrwert" von E-Learning gegeben hat. Mit dieser Debatte will ich Sie nicht langweilen. Leider sind die Effekte von E-Learning im Verhältnis zu Präsenzlernen noch immer nicht ausreichend wissenschaftlich untersucht, um klare Aussagen darüber zu 
machen. Eines lässt sich aber feststellen, dass der Großteil der heute verfügbaren E-Learning-Angebote die medialen Möglichkeiten kaum nutzt.

Ich hatte überlegt, Ihnen solche E-Learning-Angebote vorzustellen, aber meiner Ansicht nach, muss man selbst darin agieren, um sie erkunden zu können.

\section{Blended Learning}

Eine methodische Konzeption, die wir von früher als Methodenmix kennen, feiert im Blended Learning „fröhliche Urständ“. Gemeint ist damit gemixtes Lernen, das sich aus Online-Phasen und Präsenzphasen zusammensetzt. Die ersten Erfahrungen mit E-Learning haben gezeigt, dass reines Online-Lernen für viele Lernenden ein Problem darstellt, vor allem, wenn intensive Kommunikationsphasen eine Rolle spielen. Vor diesem Hintergrund bieten die Präsenzphasen die Möglichkeit den sozialen Austausch, Rückfragen und thematische Fokussierungen im direkten Kontakt vorzunehmen. Die Erfahrung zeigt, dass dadurch Online-Phasen intensiver genutzt werden.

\section{Virtual/Augmented Reality}

Technisch gestaltete dreidimensionale Lernwelten (Virtual Reality) oder Konzepte der erweiterten Realität (Augmented Reality) werden in Zukunft Lernen und Bildung verändern. Allerdings stehen wir noch am Anfang der Entwicklung, so dass sich hier noch wenig aussagen lässt. Die Technikentwickler haben allerdings den Bereich der Bildung und Kultur als einen zentralen Inhalt (Content) ausgemacht.

\section{Didaktisch-methodische Dimensionen}

Wenn Sie sich diese unterschiedlichen Lehr-/Lernarrangements anschauen, werden Sie unschwer feststellen können, dass in allen Settings differenzierte didaktisch-methodische Konzeptionen notwendig sind. Ich möchte an dieser Stelle nicht auf didaktisch-methodische Fragestellungen eingehen - vielleicht können wir das im anschließenden Workshop tun -, aber ich möchte dafür sensibilisieren, dass wir hier als Pädagog/inn/en, besonders als Medienpädagog/inn/en - auch in der Erwachsenenbildung - vor großen Herausforderungen stehen. Dies gilt auch, wenn wir uns die organisatorischen Kontexte anschauen, mit denen wir in Weiterbildungseinrichtungen konfrontiert sind.

\subsection{Organisation}

In meiner Studie "Neue Medien und Organisation in Weiterbildungseinrichtungen" (Stang 2003), die ich in diesem Jahr veröffentlicht habe, bin ich sehr ausführlich auf das Verhältnis von Neuen Medien und Organisation eingegangen. Die Fülle der Ergebnisse hier vorzustellen würde den Rahmen sprengen, aber auf ein paar Aspekte möchte ich doch hinweisen. In meiner Untersuchung habe ich allen Volkshochschulen in Deutschland knapp 1000 - einen umfangreichen Fragebogen zukommen lassen und immerhin einen Rücklauf von fast $40 \%$ gehabt. Auf der Grundlage der Auswertung habe ich fünf Einrichtungen per Clusteranalyse ausgewählt 
und mit Hilfe von Experten-Interviews untersucht. Im Überblick lassen sich folgende Aspekte besonders hervorheben:

$>$ Die technische Infrastruktur ist in den meisten Institutionen als sehr gut zu bezeichnen.

$>$ Die organisatorischen Ebenen, die für den Einsatz von Neuen Medien von besonderer Relevanz sind, sind folgende:

- Büro- und Verwaltungsalltag,

- Marketing und Öffentlichkeitsarbeit,

- Inhalte von Bildungsangeboten,

- Einsatz der Neuen Medien als unterstützendes Unterrichtsmittel,

- Gestaltung neuer Lehr-/Lernarrangements.

$>$ Derzeit werden die gravierendsten Veränderungen durch die Neuen Medien im Bereich der Verwaltung gesehen.

$>$ Der Einsatz der Neuen Medien im Angebotsbereich konzentriert sich vor allem auf den Programmbereich "Arbeit - Beruf".

$>$ Neue mediengestützte Angebotsformen wie computergestützte Selbstlernzentren und E-Learning halten nur langsam Einzug ins Programmprofil.

$>$ Die professionsadäquate Betreuung der technischen Infrastruktur durch EDV-Organisatoren ist nur selten gegeben. Oft wird die EDVOrganisation von Pädagogen realisiert.

$>$ Es fehlen übergreifende Organisationsstrukturen für die strategische Planung.

$>$ Das besondere Engagement von Mitarbeiter/innen spielt häufig eine größere Rolle als eine gezielte Entwicklungsstrategie.

$>$ Den Leiter/innen kommt eine besondere Funktion bei der Medienentwicklung in einer Einrichtung zu.

$>$ Unterschiedliche Organisationskulturen führen zu verschiedenen Strategien.

$>$ Die Organisationskultur ist wichtiger als strukturelle Rahmenbedingungen wie Finanzausstattung, Größe der Einrichtung, Personalkapazität usw.

Vielleicht können wir später in der Diskussion noch ausführlicher auf einzelne Aspekte und deren Konsequenzen eingehen.

Ein wichtiger Aspekt, den ich im folgenden beleuchten will, ist die Frage der Professionsprofile auf den unterschiedlichen Ebenen. Es ist sicher auch für Sie als Studierende von Interesse, welche Professionsdimensionen an der Schnittstelle von Medien und Erwachsenenbildung eine Rolle spielen. 


\subsection{Professionsprofile}

Wenn wir uns den Einsatz Neuer Medien in der Weiterbildung anschauen, wird deutlich, dass wir folgende Ebenen bezogen auf Professionsfragen in den Blick nehmen müssen:

$>$ Leitung,

$>$ Verwaltung,

$>$ Programmplanung,

$>$ Angebotsdurchführung und

$>$ technischer Service

\section{Leitung}

Wie ich in meiner Studie zeigen konnte, kommt der Leitung von Weiterbildungseinrichtungen im Bezug auf eine medienorientierte Organisationsentwicklung eine entscheidende Bedeutung zu. Sie entscheidet über die strategische Ausrichtung der Einrichtung, d.h. auch über die medialen Ressourcen, sowohl für die Verwaltung, als auch den pädagogischen Bereich. Innovationsmanagement wird im wesentlichen von der Leitung getragen. Hierin liegen Anforderungen für das Leitungspersonal, für die oft keine entsprechenden Strategien vorliegen.

\section{Verwaltung}

Am gravierendsten dürften sich die Arbeitsbedingungen für das Verwaltungspersonal durch den Einsatz Neuer Medien verändert haben. Wir stellen zunehmend fest, dass auch das Verwaltungspersonal stärker in Beratungszusammenhänge eingebunden ist, was neue Kompetenzprofile erfordert.

\section{Programmplanung}

Hauptberufliche pädagogische Mitarbeiter/innen sind für die Programmplanung in den Einrichtungen zuständig. Von ihnen hängt ab, in welchem Maße Neue Medien in den unterschiedlichen Programmbereichen zum Tragen kommen. Sie entscheiden über die technische Ausstattung in ihrem jeweiligen Bereich mit. Außerdem hat sich durch den Computereinsatz auch ihr Arbeitsalltag verändert. Sie übernehmen Aufgaben des Sekretariatsbereichs, wenn sie z.B. die Texte für das Bildungsprogramm direkt in den Computer schreiben. Dadurch verändert sich nicht nur das Aufgabenprofil, sondern auch das strukturelle Verhältnis von pädagogischem und Verwaltungsbereich, die heute nicht mehr in dem Maße voneinander zu trennen sind wie dies noch vor ein paar Jahren der Fall war.

\section{Angebotsdurchführung}

Die Kursleitenden sind die Schnittstelle zwischen Institution und Teilnehmenden. Von innen hängt die Gestaltung der Lehr-/Lernarrangements in besonderem Maße ab. Sie entscheiden über den Medieneinsatz, den sie allerdings nur im Rahmen der technischen Möglichkeiten der jeweiligen Einrichtung realisieren können. Von didaktisch-methodische Kompetenzen des Einsatzes von Medien in Präsenzveranstaltungen bis hin zu Beratungs- 
kompetenzen in als Teletutoren in E-Learning-Angeboten erstreckt sich das Kompetenzspektrum für Kursleitende. Die Ausdifferenzierung des pädagogischen Handels von der Lehre zur Beratung und Begleitung von Lernprozessen sowie eine veränderte Bedürfnisstruktur der Lernenden stellen dabei besondere Herausforderungen dar.

\section{Technischer Service}

Die Bedeutung des technischen Service zum Beispiel im Kontext der EDVOrganisation oder der Internet-Präsentation wurde in Weiterbildungseinrichtungen tendenziell vernachlässigt. Doch im Zusammenhang mit der Diskussion über E-Learning wird immer deutlicher, dass die Gestaltung der technischen Settings von pädagogischen Dimensionen des Bildungshandelns nicht zu trennen sind. Hier werden in Zukunft immense Herausforderungen auf Weiterbildungseinrichtungen zukommen.

Ich habe hier nur ein paar Aspekte der Professionsprofile und ihrer Veränderungen angedeutet und hoffe, dass wir dies in der Diskussion vertiefen können. Doch bevor ich zum Schluss komme, möchte ich einige kurze Anmerkungen zu den Perspektiven machen.

\section{Perspektiven}

Besonders meine letzten Ausführungen weisen auf ein Problem der Medienpädagogik hin: Fragen der organisatorischen Rahmung medienpädagogischen Handelns werden kaum als Bestandteil einer Fundierung von Medienpädagogik einbezogen.

Ein anderer Aspekt, der das Verhältnis von Medienpädagogik und Erwachsenenbildung so schwierig macht, ist die Segmentierung des medienpädagogischen Diskurses. Es gibt Expert/inn/en für Kinder oder Jugendliche, für Erwachsenen und Senioren, doch eine medienpädagogische Konzeptionierung im Bezug auf das lebenslange Lernen steht noch aus. Es gibt auch keinen Lehrstuhl in Deutschland, der sich dieser Thematik widmet. In der Entwicklung eines fundierten Konzepts an dieser Schnittstelle liegt eine große Herausforderung für die Zukunft.

Für die Pädagogische Hochschule Ludwigsburg könnte hier ein interessanter Ansatzpunkt liegen. Darüber können wir sicher im anschließenden Workshop intensiver diskutieren.

Sie werden sicher festgestellt haben, dass ich Ihnen in meinem Vortrag nur ein paar Aspekte und Perspektiven des Einsatzes Neuer Medien in der Erwachsenenbildung und zum Verhältnis von Medienpädagogik und Kommunikationskultur vorstellen konnte. Ich hoffe, dass es ausreichend war, um Ihnen einen Überblick über die aktuellen Diskurse gegeben zu haben. Ich freue mich auf die Diskussion mit Ihnen und möchte mich recht herzlich für Ihre Aufmerksamkeit bedanken. 


\section{Literatur}

Arnold, R. (2001): Selbstorganisation. In: ArNold, R./NoldA, S./NUISSL, E. (HRSG.) (2001): Wörterbuch Erwachsenenpädagogik. Bad Heilbrunn/Obb. S. 280-281.

Arnold, R./Faulstich, P./Mader, W./Nuissl von Rein, E./Schlutz, E. (2000): Forschungsmemorandum für die Erwachsenen- und Weiterbildung. Im Auftrag der Sektion Erwachsenenbildung der DGfE. Sonderbeilage zum Forschungs- und Literaturreport Weiterbildung.

BaAcke, D./Schäfer, E./Treumann, K.P./Volkmer, I. (1990): Neue Medien und Erwachsenenbildung. Berlin, New York.

BAACKE, D. (1997): Medienpädagogik. Tübingen

BALzeR, C./NUISSL, E. (HRSG.) (2000): Finanzierung lebenslangen Lernens. Bielefeld.

BAYER, M. (1998): Anforderungen an Innovationen. In: FAULSTICH, P./BAYER, M./KROHN, M. (HRSG.) (1998): Zukunftskonzepte der Weiterbildung, Projekte und Innovationen. Weinheim, München. S. 19-27.

Behrens, H./CIUPKE, P./REICHLING, N. (2001): Lernsettings in Kultureinrichtungen. In: STANG, R./PUHL, A. (HRSG.) (2001): Bibliotheken und lebenslanges Lernen. Lernarrangements in Bildungs- und Kultureinrichtungen. Bielefeld.

Bohn, H./Mikus, J./Schiemann, K. K./Seidel, G. (1987): Neue Medien - Konsequenzen für die Erwachsenenbildung. Frankfurt a.M.

BRUNS, B./GAJEWSKI, P. (2000): Multimediales Lernen im Netz. Leitfaden für Entscheider und Planer. 2. Aufl. Berlin, Heidelberg, New York.

DeUtScher BildungsRat (1970): Strukturplan für das Bildungswesen. Stuttgart.

DIETRICH, S. (2001): Selbstgesteuertes Lernen in der Weiterbildungspraxis. Ergebnisse und Erfahrungen aus dem Projekt SeGeL. Bielefeld.

DoHMEN, G. (2001): Das informelle Lernen. Hrsg. vom Bundesministerium für Bildung und Forschung. Bonn.

DRÖLL, H. (1999): Weiterbildung als Ware. Ein lokaler Weiterbildungsmarkt - das Beispiel Frankfurt. Schwalbach/Ts.

ECKeRT, A./KLIER, W./Sittenberg, G. (1983): EDV als Arbeitsinstrument für Pädagogik und Verwaltung an VHS. Planung, Anwendungsprobleme und Auswirkungen. Frankfurt a.M.

EHMANN, C. (2001): Bildungsfinanzierung und soziale Gerechtigkeit. Bielefeld.

EHSES, C./ZECH, R. (1999): Professionalität als Qualität in der Erwachsenenbildung. In: ZECH, R./EHSES, C. (HRSG.) (1999): Organisation und Lernen. Schriftenreihe für kritische Sozialforschung und Bildungsarbeit. Band 5. Hannover. S.13-57.

FAULSTICH, P. (1991): Qualitätskriterien für Erwachsenenbildung als ein Fokus der Berufsfortbildungsforschung. In: Zeitschrift für Berufs- und Wirtschaftspädagogik. Heft 7.

FAULSTICH, P. (1997): Transformationsprozesse im Institutionenspektrum der Erwachsenenbildung. In: DeRICHS-KunstMANN, K./FAulstiCH, P./TIPPELT, R. (HRSG.) (1997): Enttraditionalisierung der Erwachsenenbildung. Beiheft zum Literatur- und Forschungsreport Weiterbildung. Frankfurt a.M. S. 60-70.

FAULSTICH, P. (1998): „Selbstorganisiertes Lernen“ als Impuls für die Erwachsenenbildung. In: DeRIChS-Kunstmann et Al. (HRSg.) (1998): Selbstorganisiertes Lernen als Problem der Erwachsenenbildung. Beiheft zum Literatur- und Forschungsreport Weiterbildung. Frankfurt a.M. S. 10-13.

FAULSTICH, P./KURZWEIL, U./SchULZ-VONDERSCHER, C. (1985): Informationstechnik. Institutionen der Weiterbildung. Hrsg. von der Kontaktstelle für wissenschaftliche, künstlerische und berufliche Weiterbildung (KWW) der Gesamthochschule Kassel. Kassel.

FAULSTICH, P. ET AL. (1992): Weiterbildung für die 90er Jahre. Gutachten über zukunftsorientierte Angebote, Organisationsformen und Institutionen. Weinheim, München. 
Dr. Richard Stang: Medienpädagogik und Erwachsenenbildung

Vortrag im Rahmen des Medienpädagogischen Kolloquiums an der PH Ludwigsburg am 11.12.2003

FRIEBEL, H. ET AL. (1993): Weiterbildungsmarkt und Lebenszusammenhang. Bad Heilbrunn/Obb.

GEIßLER, H. (1998): Die Organisation pädagogischer Arbeit. In: VogEL, N. (HRSG.) (1998): Organisation und Entwicklung in der Weiterbildung. Bad Heilbrunn/Obb. S. 54-85.

GNAHS, D. (1999): Zwischenbilanz der Qualitätsdebatte. In: Literatur- und Forschungsreport Weiterbildung. Heft 43. S. 15-22.

Hagedorn, F. (1998a): Pädagogische Metamorphosen. Zum Wandel der Bildungsorganisation in der Mediengesellschaft. In: KUBICEK, H. ET AL. (HRSG.) (1998): Lernort Multimedia. Jahrbuch Telekommunikation und Gesellschaft. Heidelberg. S. 62-71.

HAGEDORN, F. (1998b): Weiterbildung als virtuelles Unternehmen? Zum Verhältnis von Multimedia und Bildungsorganisation. In: Literatur- und Forschungsreport Weiterbildung. Heft 42. S. 127-136.

KADE, J. (1997): Entgrenzung und Entstrukturierung. Zum Wandel der Erwachsenenbildung in der Moderne. In: Derichs-KunstMann, K./FAulstich, P./TIPPELT, R. (HRSG.) (1997): Enttraditionalisierung der Erwachsenenbildung. Beiheft zum Literatur- und Forschungsreport Weiterbildung. Frankfurt a.M. S. 13-31.

KERRES, M. (1998): Multimediale und telemediale Lernumgebungen. Konzeption und Entwicklung. München, Wien.

KLIMSA, P. (1993): Neue Medien und Weiterbildung. Anwendung und Nutzung in Lernprozessen der Weiterbildung. Weinheim.

KöLLINGER, P. (2001): E-Learning. Eine Marktanalyse für Deutschland. Düsseldorf.

KöRBER, K. ET AL. (1995): Das Weiterbildungsangebot im Lande Bremen. Strukturen und Entwicklungen einer städtischen Region. Bremer Texte zur Erwachsenen-Bildungsforschung 3. Bremen.

KoRING, B. (1999): Überlegungen zur didaktischen Struktur internetbasierten Lernens in der Erwachsenenbildung. In: ForSCHUNGSINSTITUT FüR ARBEITERBILDUNG (HRSG.) (1999): Jahrbuch Arbeit Kultur Bildung. Band 17. Recklinghausen. S. 9-20.

KRAUS, K. (2001): Lebenslanges Lernen - Karriere einer Leitidee. Bielefeld.

KRUG, P. (1999): Ordnungspolitische Bedeutung des Qualitätsmanagements in der Weiterbildung. In: KüCHLER, F. VON/MEISEL, K. (HRSG.) (1999): Qualitätssicherung in der Weiterbildung. Auf dem Weg zu Qualitätsmaßstäben. Frankfurt a.M. S. 20-30.

Kubicek, H./Rolf, A. (1985): Mikropolis. Mit Computernetzen in die ,Informationsgesellschaft'. Hamburg.

LANDESVERBAND DeR Volkshochschulen Niedersachsens (HRSg.) (1996): Qualitätssicherung in der Volkshochschule - Fragenkatalog zur Selbstevaluation. Hannover.

LENZEN,D./LUHMANN, N. (HRSG.) (1997): Bildung und Weiterbildung im Erziehungssystem. Lebenslauf und Humanontogenese als Medium und Form. Frankfurt a.M.

MADER, W./STANG, R. (2001): Erweitertes Schema zur Integration der Ergebnisse der Arbeitsgruppen bzw. Forschungsfelder. In: AMBOS, I./NUISSL, E. (HRSG.) (2001): Forschung zur Erwachsenenbildung. Dokumentation des Forschungsworkshops in Hofgeismar im Januar 2001. Ergänzende CD-ROM zu: AMBOS, I. (2001): Forschung zur Erwachsenenbildung. Sonderbeilage zum Literatur- und Forschungsreport Weiterbildung.

McGovern, S. (2001): European eLearning Market Forecast and Analysis, 2000-2005. Report der Firma IDC.

MEISEL, K. (1994): Marketing für Erwachsenenbildung in der Diskussion. In: MeISEL, K. ET AL. (1994): Marketing für Erwachsenenbildung? Bad Heilbrunn. S. 13-58.

MeISEL, K. (1999a): Weiterbildungsmanagement. In: TIPPELT, R. (HRSG.) (1999): Handbuch Erwachsenenbildung/Weiterbildung. 2., überarb. und aktual. Aufl. Opladen. S. 430441. 
MEISEL, K. (1999b): Dialogische Qualitätsentwicklung im Feld - Erfahrungen und Auswirkungen eines bundesweiten Projekts in der öffentlichen Erwachsenenbildung. In: KüCHLER, F. VON/MEISEL, K. (HRSG.) (1999): Qualitätssicherung in der Weiterbildung. Band 2: Auf dem Weg zu besserer Praxis. Frankfurt a.M. S. 234-254.

Meisel, K. (2001a): Marktorientierung. In: Arnold, R./NoldA, S./NuissL, E. (HRSG.) (2001): Wörterbuch Erwachsenenpädagogik. Bad Heilbrunn/Obb. S. 216-217.

MEISEL, K. (2001b): Managementprobleme in öffentlichen Erwachsenenbildungseinrichtungen. Anforderungen an intermediäre Forschungs- und Entwicklungsarbeiten für ausgewäh/te Managementaufgaben. Reihe: Grundlagen der Berufs- und Erwachsenenbildung. Band 24. Baltmannsweiler.

MeISEL, K. (2001c): Marketing. In: ARnold, R./NoldA, S./Nuisst, E. (HRSG.) (2001): Wörterbuch Erwachsenenpädagogik. Bad Heilbrunn/Obb. S. 215-216.

MeISEL, K./ROHLMANN, R./Schuldt, H.-J. (1998): Wirtschaftlichkeit in Weiterbildungseinrichtungen. Reihe: Studientexte für Erwachsenenbildung. 2., überarbeitete Aufl.. Frankfurt a.M. [1996].

NisPel, A./Stang, R./HAgedorn, F. (Hrsg.) (1998a): Pädagogische Innovation mit Multimedia. Band 1: Analysen und Lernorte. Frankfurt a.M.

Nispel, A./Stang, R./Hagedorn, F. (Hrsg.) (1998b): Pädagogische Innovation mit Multimedia. Band 2: Organisation und Fortbildung. Frankfurt a.M.

NuISSL, E. (HRSG.) (1995): Standortfaktor Weiterbildung. Bad Heilbrunn/Obb.

NUISSL, E. (1998): Leitung von Weiterbildungseinrichtungen. Studientexte für Erwachsenenbildung. Frankfurt a.M.

NUISSL, E. (1999): Ordnungsgrundsätze der Erwachsenenbildung in Deutschland. In: TIPPELT, R. (HRSG.) (1999): Handbuch Erwachsenenbildung/Weiterbildung. 2., überarb. und aktual. Aufl. Opladen. S. 389-401.

NUISSL, E. (2001): Erwachsenenbildung - Weiterbildung. In: ARNOLD, R./NOLDA, S./NUISSL, E. (HRSG.) (2001): Wörterbuch Erwachsenenpädagogik. Bad Heilbrunn/Obb. S. 8589.

NUISSL, E./REIN, A. von (1997): Corporate Identity. 2., überarb. Aufl. Frankfurt a.M. [1995].

Nuisst, E./Schiersmann, C./Siebert, H. (HRSg.) (1997): Pluralisierung des Lehrens und Lernens. Bad Heilbrunn.

Peters, S. (1999): Differenz und Kooperation von beruflicher und betrieblicher Weiterbildung. In: Forschungsinstitut für Arbeiterbildung (HRSg.) (1999): Jahrbuch Arbeit Kultur Bildung. Band 17. Recklinghausen. S. 165-180.

PetSCH, H.-J. ET AL. (1989): Allgemeinbildung und Computer. Bad Heilbrunn/Obb.

ReIn, A. von (HRSg.) (1996): Medienkompetenz als Schlüsselbegriff. Bad Heilbrunn/Obb.

ReIn, A. von (2001): Öffentlichkeitsarbeit. In: ARnold, R./NoldA, S./NuISSL, E. (HRSG.) (2001): Wörterbuch Erwachsenenpädagogik. Bad Heilbrunn/Obb. S. 241-242.

Rohlmann, R. (2001): Finanzierung. In: ARNold, R./Nolda, S./Nuisst, E. (HRSG.) (2001): Wörterbuch Erwachsenenpädagogik. Bad Heilbrunn/Obb. S. 119-120

RudoLF, K. (2001): Telelernen in der Volkshochschule. Hrsg. vom Deutschen Volkshochschul-Verband - Netzwerk Beruf und Weiterbildung. Bonn/Frankfurt a.M.

SCHÄFER, E. (2001): Auf dem Weg zum Edutainment? Medien und Erwachsenenbildung historische und aktuelle Entwicklungen. In: FriedentHAL-HAASE, M. (Hrsg.) (2001b): Erwachsenenbildung im 20. Jahrhundert - Was war wesentlich? München, Mering. S. 57-81.

SCHÄFFTER, O. (1998): Weiterbildung in der Transformationsgesellschaft. Zur Grundlegung einer Theorie der Institutionalisierung. Berlin.

SCHLÄFlI, A. (2001): Management. In: ARNold, R./NoldA, S./NUISSL, E. (HRSG.) (2001): Wörterbuch Erwachsenenpädagogik. Bad Heilbrunn/Obb. S. 212-213. 
SEIDEL, G./MIKUS, J. (1987): Überlegungen in makro- und mikrodidaktischer Sicht. In: BoHN, H./MIKUS, J./SchiemanN, K. K./SeIDEL, G. (1987): Neue Medien - Konsequenzen für die Erwachsenenbildung. Frankfurt a.M. S. 36-54.

SieBerT, H. (1999): Erwachsenenbildung in der Bundesrepublik Deutschland. Alte Bundesländer und neue Bundesländer. In: TIPPELT, R. (HRSG.) (1999): Handbuch Erwachsenenbildung/Weiterbildung. 2., überarb. und aktual. Aufl. Opladen. S.54-80.

STANG, R. (1998): Organisationen im Umbau. Anforderungen an Weiterbildungsinstitutionen. In: NisPel, A./Stang, R./HAgedorn, F. (Hrsg.) (1998b): Pädagogische Innovation mit Multimedia. Band 2: Organisation und Fortbildung. Frankfurt a.M. S. 21-24.

StANG, R. (1999): Qualität Kultureller Bildung. In: KüCHLER, F. VON/MeISEL, K. (HRSG.) (1999): Qualitätssicherung in der Weiterbildung. Auf dem Weg zu Qualitätsmaßstäben. Frankfurt a.M. S. 46-55.

StANG, R. (2001a): Neue Medien in der Erwachsenenbildung. In: STANG, R. (HRSG.) (2001c): Lernsoftware in der Erwachsenenbildung. Bielefeld. S. 13-22.

StANG, R. (2001b): Anforderungen an zukünftige Lernarrangements. In: STANG, R./PUHL, A. (HRSG.) (2001): Bibliotheken und lebenslanges Lernen. Lernarrangements in Bildungs- und Kultureinrichtungen. Bielefeld. S. 24-31.

StANG, R. (HRSG.) (2001c): Lernsoftware in der Erwachsenenbildung. Bielefeld.

STANG, R. (2001d): Was steckt hinter dem neuen Schlagwort ,E-Learning'? In: Beilage Weiterbildung der Frankfurter Rundschau vom 22.9.2001.

STANG, R. (2002): Vernetzung als Zukunftsmodell. Zur Kooperation von Kultur- und Bildungsinstitutionen. In: PUHL, A./STANG, R. (HRSG.) (2002): Bibliotheken und die Vernetzung des Wissens. Bielefeld. S. 13-19.

StANG, R. (2003): Neue Medien und Organisation in Weiterbildungseinrichtungen. Anregungen für eine medienorientierte Organisationsentwicklung. Bielefeld.

Stang, R./Apel, H./Hagedorn, F. (Hrsg.) (1999): Pädagogische Innovation mit Multimedia. Band 3: Konzepte, Analysen, Perspektiven. Frankfurt a.M.

Stang, R./Nuissl, E./Apel, H./Kraft, S./Möller, S. (2001): Neue Medien und lebenslanges Lernen. In: ARBeitsStab Forum BILdUNG (HRSG.) (2001): Lernen - ein Leben lang. Vorläufige Empfehlungen und Endbericht. Materialband 9. Bonn. S. 170-184.

STRUNK, G. (2000): Erwachsenenbildung als Teil des öffentlich verantworteten Weiterbildungsbereichs. In: Hessische Blätter für Volksbildung. Heft 4. S. 317-329.

TIPPELT, R./ECKERT, T./BARZ, H. (1996): Markt und integrative Weiterbildung. Zur Differenzierung von Weiterbildungsanbietern und Weiterbildungsinteressen. Bad Heilbrunn/Obb.

UMLAUF, K. (2001): Die öffentliche Bibliothek als Lernort. In: STANG, R./PuHL, A. (HRSG.) (2001): Bibliotheken und lebenslanges Lernen. Lernarrangements in Bildungs- und Kultureinrichtungen. Bielefeld. S. 35-55.

WEINBERG, J. (2000): Einführung in das Studium der Erwachsenenbildung. Überarbeitete Neuaufl. Bad Heilbrunn/Obb.

Wittpoth, J. (2001): Medien. In: ARnold, R./NoldA, S./Nuisst, E. (HRSg.) (2001): Wörterbuch Erwachsenenpädagogik. Bad Heilbrunn/Obb. S. 220-222.

WolPERT, A. (1998): VHS goes online. Ergebnisse einer Befragung. In: NISPEL, A./STANG, R./HAgedorn, F. (HRSG.) (1998a): Pädagogische Innovation mit Multimedia. Band 1: Analysen und Lernorte. Frankfurt a.M. S. 98-106.

ZEIDLER, B. (2001): Lernsoftware für das Sprachenlernen. In: STANG, R. (HRSG.) (2001c): Lernsoftware in der Erwachsenenbildung. Bielefeld. S. 41-58. 